\title{
La motivation en formation : une dimension réhabilitée dans un environnement d'apprentissage en mutation
}

\author{
Motivation and education : a rehabilitated concept \\ in a changing learning environment
}

Thierry PELACCIA', Hervé DELPLANCQ', Emmanuel TRIBY², Cécile LEMAN ${ }^{1}$, Jean-Claude BARTIER ${ }^{1}$, Jean-Pierre DUPEYRON ${ }^{3}$

\begin{abstract}
Messages clés • L'environnement d'apprentissage des étudiants des filières de santé est en mutation dans un contexte d'usage croissant de méthodes d'enseignement actives et d'introduction massive des technologies de l'information et de la communication. - Dans cet environnement incitant l'étudiant à simpliquer activement dans la construction de ses connaissances, la question de la motivation - c'est-à-dire des facteurs à l'origine de son engagement en formation et de son investissement dans les activités d'apprentissage - devient centrale. $\bullet$ La motivation a fait l'objet d'études sous l'angle de multiples cadres conceptuels, influencés par les paradigmes successifs de la psychologie et des sciences de l'apprentissage. $\bullet$ Les théories récentes, qui sappuient notamment sur les perspectives des sciences cognitives, et les travaux de recherche réalisés dans ce champ d'étude autorisent à formuler un certain nombre de recommandations permettant aux concepteurs et aux responsables de dispositifs de formation de prendre en compte cette dimension majeure de la réussite.
\end{abstract}

Mots clés Motivation ; pédagogies actives ; technologies de l'information et de la communication ; sciences cognitives ; autodétermination ; compétence.

Key messages • The learning environment of health sciences students is changing in the context of the growing use of active-based teaching methods and also in the context of the massive introduction of technologies of information and communication. - In this changing learning environment, which encourages students to be actively involved in the construction of their knowledge, the question of motivation - that is factors that cause educational self-commitment and investment in learning activities - becomes central. $\bullet$ Motivation as been studied from different conceptual frameworks, which are influenced by successive paradigms of psychology and learning sciences. $\bullet$ Recent theories, which are supported by cognitive sciences concepts and research works completed in this field of study, authorize to set out a number of recommendations allowing educational designers and project managers to take into account this major dimension of success.

Key words Motivation; active-based education; technologies of information and communication; cognitive sciences; self-determination; competence. .

Pédagogie Médicale 2008;9:103-21

1- Service d'aide médicale urgente (SAMU 67) et Centre d'enseignement des soins d'urgence (CESU 67) - Hôpitaux universitaires de Strasbourg.

2- Faculté de psychologie et des sciences de l'éducation - Département des sciences de l'éducation - Université Louis Pasteur de Strasbourg.

3- Pôle d'anesthésie - réanimation chirurgicale - SAMU/SMUR - Hôpitaux universitaires de Strasbourg.

Correspondance: Thierry PELACCIA. SAMU 67 - CESU 67, Hospices civils, 1 place de l'hôpital, 67000 Strasbourg, France.

Mailto:thierry.pelaccia@wanadoo.fr 


\section{Références}

\section{Introduction}

L'utilisation privilégiée de méthodes d'enseignement sollicitant explicitement l'activité des étudiants et le recours accru, de leur part, aux technologies de l'information et de la communication au cours des formations aux professions de santé ont transformé le statut de l'apprenant. L'étudiant, souvent passif dans le cadre d'un dispositif conventionnel de formation, et dont les connaissances étaient élaborées à partir d'un savoir mis à disposition par l'enseignant sur un mode expositif, après mise en forme didactique, devient l'élément central de la construction de ses compétences. Un tel rôle actif, apprécié par certains, source de difficultés et d'échecs pour d'autres, conduit à s'interroger sur la motivation en tant que facteur désormais incontournable de l'apprentissage, alors qu'elle a été pendant longtemps occultée dans le cadre d'une réflexion ingénierique qui était surtout focalisée sur le demandeur et sur le prestataire.

La réhabilitation des attentes et des projets individuels de l'étudiant dans le dispositif de formation qui lui est destiné pose en effet la question des « ressources motivationnelles " qu'il mobilise pour s'engager dans ses activités d'apprentissage et pour gérer toutes les interactions qui y sont reliées.

L'identification et la compréhension des facteurs qui influencent à la fois la décision d'entrer en formation et les différents comportements et approches qui se manifestent à l'égard de l'apprentissage constituent pour les enseignants une ressource majeure lors de la conception d'un dispositif de formation et lorsqu'il s'agit de réfléchir à ses évolutions. Dans cette perspective, nous proposons dans un premier temps une définition du concept de motivation, puis une description approfondie des théories actuelles et des travaux de recherche réalisés dans ce champ d'étude dans le cadre de la formation des professionnels de santé. La dernière partie de la contribution sera consacrée à la formulation de propositions concrètes permettant d'intégrer cette dimension majeure de l'apprentissage dans la conception et dans l'amélioration des dispositifs de formation initiale et continue.

\section{Définition du concept}

Vallerand et Thill ${ }^{1}$ définissent la motivation comme "le construit hypothétique utilisé afin de décrire les forces internes etlou externes produisant le déclenchement, la direction, l'intensité et la persistance du comportement". Le fait d'assimiler la motivation à un phénomène dont on postule l'existence, mais qu'on ne peut qu'inférer à partir de certains comportements, traduit le caractère théorique et abstrait d'une notion qui a fait l'objet d'efforts multiples et divergents de conceptualisation.

Cette définition exprime également le consensus actuel autour d'une conception de la motivation ayant des origines à la fois intrinsèques et extrinsèques et résultant d'un processus qui régule les aspects dynamiques $\mathrm{du}$ comportement humain en termes de variables, telles que la quantité d'énergie investie ou la durée.

Un tel cadre conceptuel est donc par définition extrêmement vaste et peut être traité à deux niveaux ${ }^{2,3}$, selon que l'on considère la motivation en formation comme un ensemble de variables dirigeant le comportement ou bien le dynamisant :

- il s'agit, d'une part, des raisons qui poussent l'individu à s'inscrire à une formation donnée (par exemple les études de médecine). Dans cette approche, la motivation est en quelque sorte le sens que va donner l'individu à son action et nous parlerons par la suite de motifs d'engagement ;

- il s'agit d'autre part, du déclenchement des pratiques d'apprentissage et de l'investissement et de la persévérance dans les tâches entreprises une fois l'entrée en formation effective. Nous parlerons par la suite de dynamique motivationnelle.

A l'issue d'un parcours historique des théories de la motivation, nous consacrerons une partie de ce texte à chacune de ces dimensions, bien que la distinction des deux niveaux d'analyse puisse paraître artificielle et qu'un certain nombre de modèles théoriques présentés par la suite offrent une approche indifférenciée du concept.

\section{Les théories historiques de la motivation}

Si la motivation est aujourd'hui reconnue comme un facteur psychologique déterminant en situation d'apprentissage ${ }^{4}$, les courants dominants successifs de la pédagogie n'y accordèrent pas un intérêt égal.

Deux approches opposées du concept dominèrent le paysage de la psychologie jusque dans les années soixante ${ }^{5}$ :

- le premier paradigme repose sur l'idée que la motivation relèverait uniquement de facteurs individuels et 
qu'il s'agirait avant tout d'un trait de personnalité : on est motivé ou non, de la même façon que l'on peut être réservé ou audacieux ;

- la seconde approche considère que la motivation est liée à des facteurs environnementaux sur lesquels il suffirait d'agir pour obtenir l'effet souhaité.

\section{L'hédonisme}

Les philosophes grecs pensaient que les comportements humains étaient dictés par la passion ou encore par la recherche du bien-être. L'hédonisme concevra ainsi la conduite des individus dans une optique personnelle et rationnelle visant à privilégier les comportements source de plaisir et à éviter ceux qui génèrent de l'insatisfaction ou de la frustration.

La limite de cette approche se situe dans le caractère subjectif et donc difficilement accessible du plaisir qu'il est impossible d'opérationnaliser.

\section{Les approches biologiques}

Les premières études sur la motivation, réalisées au début du $\mathrm{XX}^{\mathrm{e}}$ siècle par McDougall, furent associées aux instincts, c'est-à-dire à des comportements innés, biologiquement préétablis et essentiels à la survie . Cette approche domina la psychologie du début du $\mathrm{XX}^{\circ}$ siècle, très empreinte des cadres conceptuels de la physique et de la biologie.

Elle sera notamment exploitée dans les années trente par le biologiste américain Cannon, qui voyait dans nos conduites l'accomplissement d'un besoin de satisfaire l'équilibre physiologique de l'organisme.

\section{Les approches béhavioristes (ou comportementalistes)}

L'approche béhavioriste s'oppose à la conception des comportements en termes d'instincts. Elle domina le champ de la psychologie de la motivation jusque dans les années soixante.

Pavlov et Watson sont les pionniers d'un courant de pensée qui situe l'environnement au cœur de l'explication des conduites humaines et qui considère que l'apprentissage repose sur la modification des comportements observables en réponse à des stimuli externes ${ }^{7}$. Dans le concept de conditionnement opérant, initié par Thorndike au début du XX et popularisé dans les années trente par Skinner, nos conduites sont dictées par l'expérience des conséquences qu'elles ont provoquées et qui rendent plus ou moins probable que le comportement se produise à nouveau. Cette approche n'est pas sans rappeler celle de l'hédonisme. Toutefois, la recherche de satisfaction est ici la conséquence $a$ posteriori de stimuli externes produisant un renforcement positif ou négatif sur les comportements.

Pour Hull, l'étude des comportements nécessite de prendre en compte l'existence de variables intermédiaires entre le stimulus et la réponse, qui n'avaient pas fait l'objet d'une attention particulière de la part de ses prédécesseurs, malgré des observations expérimentales contradictoires ${ }^{7}$. Il s'agit en particulier de la force de l'habitude et du niveau des besoins, qui s'inscrivent dans une vision homéostatique des comportements générés par un déficit ou par une rupture de l'équilibre interne et par la recherche d'un retour à l'état antérieur. La motivation - les drives dans le modèle de Hull - fera l'objet de la première formulation théorique.

Le béhaviorisme laissera une empreinte très marquée dans notre système éducatif, en particulier dans les structures de formation initiale, dont les dispositifs d'évaluation reposent - consciemment ou non - largement sur ces théories.

\section{Critique des théories historiques de la motivation}

Les modèles homéostatiques de la motivation seront remis en cause lorsque les progrès réalisés par la science permirent de démontrer que l'état naturel des cellules des organismes vivants est actif et non inerte. En d'autres termes, l'individu n'est jamais passif, même en l'absence de stimuli externes et de besoins. Il se comporte comme un être autonome, capable de rompre les équilibres préexistants et de transformer le milieu dans lequel il évolue, afin d'y réaliser ses projets.

C'est l'absence d'intérêt pour cet aspect de la motivation qui fut à l'origine des plus vives critiques à l'égard de l'approche béhavioriste, qui réduisait volontairement ses observations aux seuls comportements visibles, mesurables et quantifiables. Ayant délibérément mis de côté les caractéristiques psychiques, différenciant l'animal de l'homme et assurant la supériorité fonctionnelle de ce dernier, la perspective béhavioriste occultait de fait l'existence d'une médiation cognitive des stimuli par le sujet et la dimension intentionnelle des comportements. Ces caractéristiques demeureront ainsi cloisonnées dans une «boîte noire » - le cerveau -, à laquelle les béhavioristes s'interdisaient d'accéder. Le modèle théorique de Hull amorcera la transition vers une approche constructiviste de la motivation, qui n’a depuis lors cessé de s'affirmer au détriment des conceptions historiques. 


\section{Références}

La fin de l'hégémonisme du béhaviorisme (comportementalisme) ouvrira la voie à des dizaines de modèles théoriques de la motivation, dont nous exposons ici les plus pertinents au regard de notre champ d'étude et des courants de pensée actuellement dominants.

\section{Le renouveau cognitiviste}

La réhabilitation de la dimension cognitive dans l'explication causale de nos comportements ${ }^{8}$ a profondément bouleversé la vision des rapports du sujet à son environnement.

De passif, dirigé par des forces externes ou internes visant à préserver un équilibre ou à générer du plaisir, l'individu devient un être pensant capable d'autodétermination et la motivation est conçue comme "une structure cognitivo-dynamique dirigeant l'action vers des buts concrets $"^{6}$.

Les développements ultérieurs de notre travail s'appuient résolument sur une telle perspective cognitiviste de la motivation, en distinguant les deux niveaux d'analyse énoncés plus haut : les motifs d'engagement en formation et la dynamique motivationnelle.

\section{Les motifs d'engagement en formation}

Dans cette partie, nous développons les facteurs à l'origine d'une démarche individuelle d'entrée en formation, telle que l'inscription en première année de médecine, le choix de la spécialité ou encore la participation à une action d'éducation permanente ciblée sur la prise en charge de l'arrêt cardiaque. Au regard de cette problématique, la distinction des contributions respectives des déterminants intrinsèques et de ceux ayant une origine extérieure à l'individu est une approche pertinente, qui a servi de modèle de recherche dominant depuis la fin des années soixante-dix?. La perspective mécanique du paradigme béhavioriste niait cette double contribution, alors que les conceptions interactionnistes des cognitivistes postulent les interactions réciproques de ces deux champs.

\section{La motivation intrinsèque}

La motivation intrinsèque est considérée comme le moteur de la réalisation d'un acte dont l'individu retire du plaisir ou de la satisfaction ${ }^{10}$. Si l'on considère, comme Nuttin ${ }^{6}$, qu'un comportement est une forme d'entrée en relation physique ou symbolique avec un objet, le but intrinsèque d'une activité est un moyen "d'approfondir ou d'intensifier " cette "relation avec l'objet ». Certains auteurs conçoivent de ce fait ces compor- tements comme des actes non motivés, dans la mesure où ils s'accomplissent spontanément. La dimension motivationnelle n'apparaîtrait dans l'absolu que lorsqu'on tenterait d'entraver leur occurrence. Pour Nuttin, au contraire, ces comportements sont fortement motivés, notamment du fait que les activités réalisées sont perçues comme un outil essentiel au service du développement personnel.

Le besoin d'autodéveloppement, le besoin d'accomplissement, mais aussi les conceptions de soi et de son environnement d'apprentissage, ainsi que le rapport de l'individu au savoir, sont intimement liés à la notion de motivation intrinsèque en formation.

\section{Le besoin conscient de développement autonome}

Il pousse l'individu à se construire des buts nouveaux afin de tendre vers le progrès et d'aller au-delà de ce qu'il est déjà parvenu à réaliser ${ }^{11}$. L'inscription à l'université ou, d'une façon générale, à une formation de l'enseignement supérieur peut permettre de réaliser ce besoin de développement. Comme le souligne Nuttin ${ }^{6}$, les dispositifs de formation continue sont également "des institutions qui résultent de l'élaboration cognitive $d u$ besoin humain vers le progrès".

\section{Le besoin d'accomplissement}

C'est un concept développé par les psychologues américains McClelland et Atkinson dans les années cinquante $^{12}$. Il explique l'existence de forces orientant les comportements de l'individu vers des activités le menant au succès et lui permettant d'éviter les échecs. Les effets recherchés sont en rapport avec le besoin de compétence, qui pousse le sujet à démontrer son habileté au regard de lui-même ou des autres. Le fait de rivaliser et de surpasser autrui devient alors un enjeu essentiel en termes de reconnaissance sociale et d'image de soi. Nous reviendrons sur le besoin d'accomplissement dans le chapitre consacré à la dynamique motivationnelle.

\section{Les conceptions de soi et de son environnement}

Ce sont des images construites autour de représentations cognitives dans lesquelles l'individu va se projeter. L'étudiant marqué dans son histoire par un événement à l'origine d'une décision ferme d'exercer une profession de santé inscrit ses ambitions dans le cadre de ce que Nuttin appelle la "force motivationnelle des idéologies". Il en serait de même des individus qui s'engagent dans une filière de formation aux métiers de la santé car ils n'envisagent leur satisfaction professionnelle qu'à travers la dimension humaniste et philanthrope associée à 
leur futur métier. Dans le domaine de la formation continue, la question de la dynamique identitaire professionnelle est ici centrale ${ }^{2}$. Par exemple, la maîtrise des " gestes qui sauvent " par les soignants participant à une formation ciblée sur la prise en charge de l'arrêt cardiaque est associée à un enjeu identitaire fort, socialement valorisé, dont l'affirmation ou la réaffirmation constituent une source importante de motivation chez ceux qui considèrent qu'elle fait partie des compétences que doit posséder tout professionnel de santé.

\section{Le rapport au savoir}

C'est un construit singulier reposant sur le "rapport an monde, à l'autre et à soi-même d'un sujet confronté à la nécessité d'apprendre $»^{13}$. Il dépend du vécu de l'individu qui se forge une représentation de la tâche à accomplir autour de valeurs, d'opinions ou encore de croyances. Selon Charlot ${ }^{13}$, cette notion nouvelle dans le champ des sciences humaines a pour intérêt de révéler une dimension novatrice du savoir "comme sens et plaisir". Il s'agit du plaisir d'apprendre, du plaisir de savoir ou encore de la satisfaction à surmonter des difficultés.

\section{La motivation extrinsèque}

La motivation extrinsèque dépend de facteurs extérieurs au sujet. Nuttin ${ }^{6}$ la définit comme la poursuite par l'individu de buts distincts des objectifs propres de l'activité déployée pour y parvenir. La motivation extrinsèque pourrait ainsi trouver sa source dans l'octroi direct ou indirect de récompenses (tels que l'obtention d'un diplôme, d'une promotion, ou encore l'accès à des loisirs à un prix réduit grâce à l'acquisition du statut d'étudiant) ou dans l'évitement de sanctions (telle que la suppression d'une prime ou du soutien financier de la part des parents). L'inscription dans une filière de l'enseignement supérieur dans l'optique d'une promotion sociale ou pour s'éloigner intentionnellement de l'environnement familial s'inscrit également dans le cadre de la motivation extrinsèque.

\section{La théorie de l'autodétermination}

Deci et $\operatorname{Ryan}^{10}$ ont proposé une approche de la motivation basée sur le niveau d'autodétermination. Selon leur théorie, trois besoins psychologiques sont à la base de la motivation humaine : le besoin d'autonomie, le besoin de compétence et le besoin d'appartenance sociale. Deci et Ryan estiment que le besoin d'autonomie est le plus fondamental dans l'explication de nos choix. Il s'agit, pour l'individu qui s'engage dans une activité, de percevoir qu'il est seul à l'origine de ses comportements?
Nous parlerons ainsi par la suite de motivation autodéterminée lorsque le besoin d'autonomie est satisfait.

Deci et Ryan ${ }^{14}$ positionnent la motivation intrinsèque au sommet d'une échelle graduée par le niveau d'autodétermination. Ils situent à l'autre extrémité les individus manifestant des comportements qu'ils ne parviennent pas à mettre en relation avec leurs conséquences ; "l'amotivation" désigne ainsi les étudiants inscrits à une formation sans réellement en comprendre la raison et l'intérêt.

Entre l'amotivation et la motivation intrinsèque, les deux psychologues américains distinguent trois types de facteurs de régulation générant des comportements extrinsèquement motivés :

La régulation externe correspond à la définition communément admise de la motivation extrinsèque, telle que nous l'avons précédemment exposée. Les récompenses et les sanctions sont les principaux facteurs de régulation susceptibles de générer des comportements d'apprentissage dictés, par exemple, par la volonté des parents ou d'un supérieur hiérarchique. Leur suppression tend à annihiler la motivation du sujet.

La régulation introjectée provoque des comportements dictés par la représentation que se fait l'individu de leurs conséquences dans un registre psychoaffectif. Il peut s'agir : - d'un sentiment de culpabilité ou de honte en cas de non accomplissement de l'activité, en raison, par exemple, de la pression sociale exercée par l'environnement familial ou professionnel. Mucchielli ${ }^{15}$ parle de contrôle social subjectif en désignant "la capacité d'un groupe social à rendre effectives ses normes et ses règles " et "à faire en sorte qu'elles soient appliquées par ses membres". Le fait de s'inscrire en première année de médecine ou de privilégier l'internat de chirurgie par crainte d'être mal vu par son entourage familial, constitué respectivement d'une lignée de médecins ou de chirurgiens, relève ainsi de la motivation extrinsèque par régulation introjectée. Il en est de même des soignants se formant aux techniques de réanimation cardio-pulmonaire parce qu'ils appréhendent le regard critique de leur entourage professionnel désapprouvant leur manque manifeste d'intérêt pour un outil susceptible de sauver des vies ;

- d'un sentiment de fierté lié, par exemple, à l'exercice d'une profession associée à une forte dimension humaniste ou à la maitrise des " gestes qui sauvent ", dans l'optique de valoriser son image. Ces comportements sont faiblement autodéterminés, dans la mesure où l'in- 


\section{Références}

dividu n'a pas le sentiment de choisir librement l'activité dans laquelle il s'engage.

La régulation identifiée se caractérise par la reconnaissance d'une valeur instrumentale aux comportements adoptés ; le sujet va participer à une activité parce qu'elle est importante pour lui, même si elle n'est pas nécessairement intéressante. La formation devient alors l'instrument de la réalisation des projets personnels ou professionnels de l'individu.

Le fait de s'engager dans une filière de l'enseignement supérieur dans le but d'exercer un métier prestigieux ou d'avoir une activité libérale relève de la motivation extrinsèque par régulation identifiée. Il en est de même des professionnels de santé se formant aux gestes et aux soins d'urgence pour être capable de porter assistance à leurs proches en cas de nécessité.

Deci et Ryan soulignent que la nature des comportements adoptés par les individus résulte souvent d'expériences passées qui forgeront notamment la perception d'autonomie et de compétence ${ }^{16}$, mais également de facteurs environnementaux et contextuels ${ }^{17}$.
L'analyse des comportements d'apprentissage selon ce modèle fondé sur l'existence d'un continuum entre amotivation, motivation extrinsèque et motivation intrinsèque - liés par un niveau croissant d'autodétermination - a fait l'objet de plusieurs travaux confirmant son intérêt dans les domaines de l'éducation et de la formation $^{9,18}$. Elle a ainsi été adoptée dans de nombreuses recherches qui ont notamment permis de mettre en évidence la corrélation entre le degré d'autodétermination et les conséquences positives en termes d'implication, de flexibilité cognitive, de persévérance et de performance dans les activités entreprises, notamment celles liées à l'apprentissage ${ }^{14}$.

Deci et Ryan recommandent de ce fait de promouvoir des activités et des techniques pédagogiques susceptibles d'entretenir ou de générer des comportements fortement autodéterminés, c'est-à-dire ayant une origine intrinsèque ou extrinsèque par régulation identifiée.

La théorie de l'autodétermination (figure 1) a également pour intérêt majeur de ne pas considérer la motivation comme un phénomène unitaire et immuable, mais au contraire inconstant et variable, tant sur le plan qualitatif que quantitatif ${ }^{14}$.

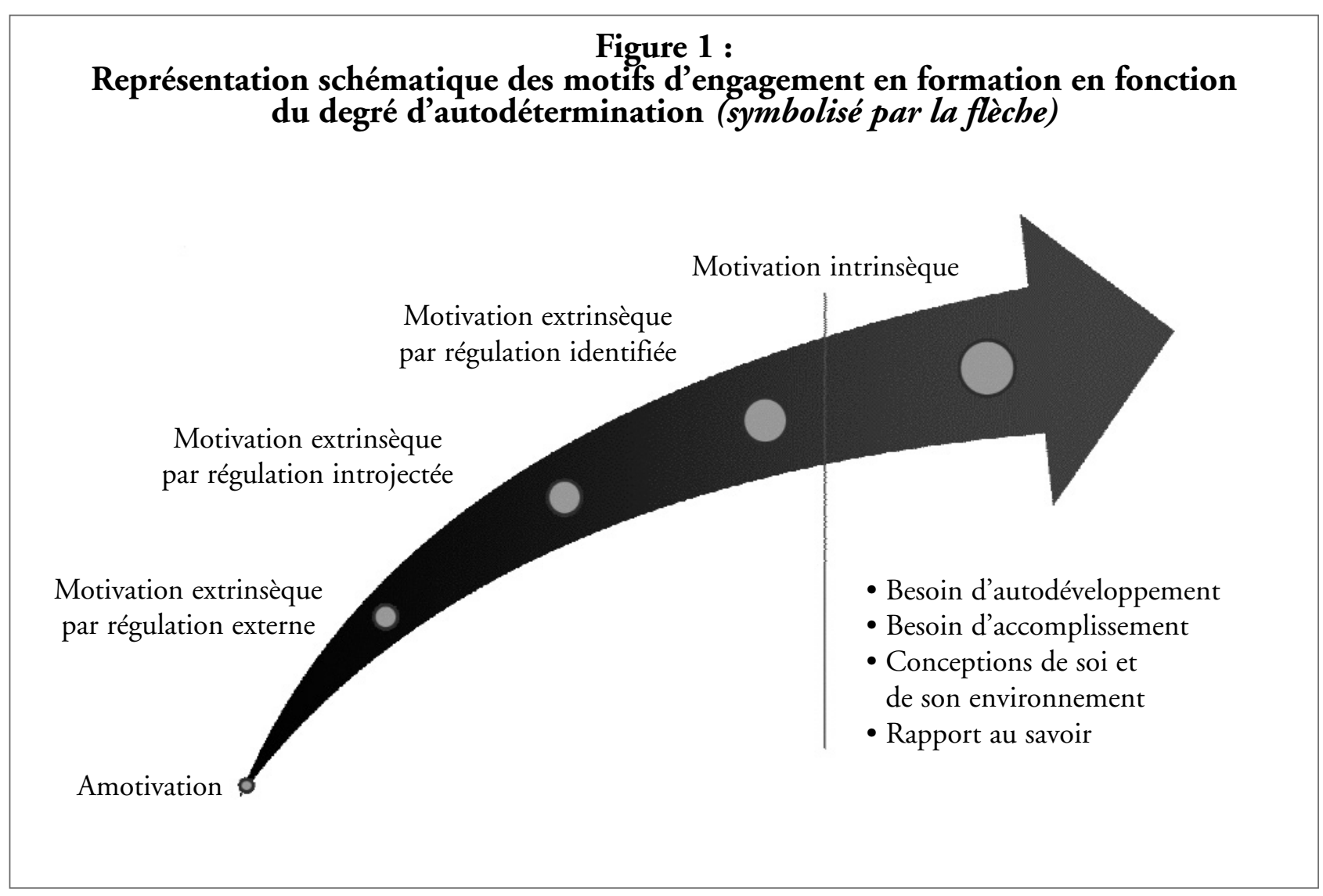


Certains facteurs, notamment contextuels, susceptibles d'influencer la nature et le niveau motivationnels deviennent alors aisément modifiables, ce qui ouvre des perspectives intéressantes en matière de formation.

\section{La dynamique motivationnelle}

Nous appellerons dynamique motivationnelle, le déclenchement des pratiques d'apprentissage et l'évolution du profil motivationnel des étudiants une fois l'entrée en formation effective, déterminant ainsi leur degré d'investissement et de persévérance dans les actions entreprises.

La théorie de l'autodétermination et la distinction traditionnelle entre motivation intrinsèque et extrinsèque sont importantes à considérer dans cette perspective d'étude. L'usage de récompenses extrinsèques, héritage du béhaviorisme, est omniprésent dans les milieux scolaires et universitaires (attribution de notes et de mentions, classements, distribution de prix aux meilleurs étudiants, etc.), ainsi que dans le monde du travail (obtention de promotions et de primes, revalorisations salariales, etc.). Elle repose sur l'idée communément admise et partagée que ces récompenses ont un impact positif sur la motivation des individus auxquels elles sont destinées.

\section{La théorie de l'évaluation cognitive}

La théorie de l'évaluation cognitive est une sous-théorie de la théorie de l'autodétermination, développée dans les années quatre-vingt par Deci et Ryan ${ }^{10}$. Elle cherche précisément à déterminer l'influence des récompenses externes sur la motivation intrinsèque. Deci et Ryan ${ }^{19}$ considèrent ainsi que ce ne sont pas les facteurs contextuels en euxmêmes qui déterminent le comportement, mais plutôt la signification que l'individu leur accorde au regard de la perception de compétence et d'autonomie qu'ils génèrent ; les facteurs extrinsèques qui augmentent la perception d'autonomie majoreront la motivation intrinsèque; de la même façon, les facteurs extrinsèques qui augmentent la perception de compétence - dans la mesure où ils sont accompagnés d'une perception d'autonomie - auront un impact positif sur la dynamique motivationnelle. L'importance des interactions entre l'apprenant et son environnement d'apprentissage a également été soulignée par Paris et Turner dans leur concept de « motivation située $»^{20}$.

La politique managériale dans le monde du travail, ainsi que les orientations pédagogiques et évaluatives dans les milieux de la formation sont donc déterminantes dans l'influence des récompenses externes sur la motivation. Deci et Ryan ${ }^{19}$ distinguent ainsi deux types de récompenses : les récompenses tangibles (telles que les mentions ou les primes) et verbales (tel qu'un feedback positif suite à l'exécution correcte d'une tâche). Ils leur attribuent deux fonctions possibles : une fonction contrôlante, lorsque la récompense est perçue comme une forme de surveillance du comportement et qu'elle encourage la compétition, et une fonction informationnelle, lorsqu' elle est perçue comme visant à renseigner l'individu sur son niveau de compétences et qu'elle encourage l'autonomie.

Les études inspirées de cette approche sont nombreuses et les résultats divergent quant à l'influence des récompenses externes sur la motivation intrinsèque. Une méta-analyse, réalisée en 1999 par Deci et al..$^{21}$ et portant sur 128 travaux de recherche, confirme la pertinence de la théorie de l'évaluation cognitive quant à la dimension non-unitaire des récompenses externes au regard de la motivation. Ainsi, les récompenses verbales, lorsqu'elles sont formulées dans un contexte informationnel, tendent à majorer la motivation intrinsèque, puisqu'elles augmentent la perception de compétence. Il s'agit, selon les auteurs, de la seule situation dans laquelle la motivation intrinsèque peut être renforcée par des facteurs externes. A l'opposé, les récompenses tangibles diminuent le niveau motivationnel intrinsèque, ceci d'autant plus que l'activité était initialement perçue comme intéressante. Dans cette situation, l'individu aura, en effet, tendance à rattacher ses efforts à des causes purement externes, liées à la perspective, par exemple, d'être bien classé au sein de sa promotion. Dès lors, ces récompenses seront perçues par l'individu comme un moyen de contrôler son activité et affecteront ainsi ses besoins d'autonomie et de compétence.

Cet effet préjudiciable des récompenses tangibles n'apparait bien entendu que lorsque celles-ci sont annoncées ${ }^{21}$. Dans le cas contraire, la motivation n'est, en effet, pas affectée, l'individu n'ayant pas le sentiment que ses comportements sont guidés par la recherche d'un bénéfice secondaire, puisque celui-ci n'était pas attendu.

Les implications pratiques de ces études en matière d'éducation et de formation sont majeures et tendent à remettre largement en cause l'utilisation des récompenses externes, telles qu'elles sont conçues dans la plupart de nos systèmes scolaires et universitaires. Nous y reviendrons dans les recommandations formulées dans la dernière partie du document.

\section{La théorie de l'attribution causale}

Cette approche de la motivation fut développée par le psychosociologue américain Weiner dans les années 


\section{Références}

quatre-vingt ${ }^{22,23}$. Le postulat de départ repose sur le fait que les individus recherchent une origine à tout ce qui leur arrive, qu'il s'agisse d'événements positifs ou non. Ainsi, en situation de formation, l'apprenant pourra attribuer ses succès et ses échecs à lui-même (on parle " d'auto-attribution ") ou à autrui (on parle "d'hétéroattribution $»)$.

De façon plus précise, Weiner propose de prendre en considération trois dimensions dans la réaction d'un apprenant face à ses succès et à ses échecs :

- le lieu de la cause distingue les causes internes à l'individu (les aptitudes intellectuelles, les stratégies utilisées lors des révisions, le manque de travail, la fatigue) des causes externes (la chance, la recherche de récompenses, les conditions d'examen, le niveau de difficulté des sujets) ;

- la stabilité de la cause fait écho à sa temporalité : elle peut être stable, lorsque l'apprenant la considère comme permanente (les aptitudes intellectuelles) ou modifiable, lorsqu'elle est susceptible d'évoluer dans le temps (le manque de travail) ;

- la contrôlabilité situe la responsabilité de l'individu dans l'événement : la cause peut être contrôlable (le manque d'efforts, la fatigue liée à un manque de repos) ou incontrôlable (la sévérité du professeur, le fait d'avoir été malade le jour de l'examen).
L'impact des attributions causales sur la motivation est majeur, dans la mesure où leur nature influencera l'estime personnelle et par conséquent la perception de compétence, de même que la perception d'autonomie dont nous avons précédemment souligné l'importance. Ainsi, l'attribution d'un succès à une cause interne et stable (telles que les aptitudes intellectuelles) augmente le niveau de confiance en soi et la perception d'autonomie, en particulier lorsqu' elle est contrôlable (telles que les stratégies d'apprentissage). En revanche, une attribution causale similaire en cas d'échec diminue la perception de compétence, en particulier lorsque celle-ci est perçue comme incontrôlable (le manque d'aptitudes intellectuelles).

Il y a un lien étroit entre l'attribution causale et la motivation intrinsèque; l'individu ne développera, en effet, un profil motivationnel fortement autodéterminé que dans la mesure où il considère que ses échecs et ses réussites sont liés à des causes contrôlables. Par ailleurs, sa perception d'autonomie sera majorée par l'attribution de ses réussites et de ses échecs à des causes internes.

Le concept d'attribution causale (tableau 1) est proche de celui de locus de contrôle développé par Rotter dans les années soixante ${ }^{24}$.

\begin{tabular}{|c|c|c|c|c|}
\hline \multicolumn{5}{|c|}{$\begin{array}{l}\text { Tableau 1: } \\
\text { Exemples d'attributions causales }\end{array}$} \\
\hline \multirow{2}{*}{$\begin{array}{l}\text { Attribution } \\
\text { causale }\end{array}$} & \multicolumn{2}{|c|}{ Interne } & \multicolumn{2}{|c|}{ Externe } \\
\hline & contrôlable & non contrôlable & contrôlable & non contrôlable \\
\hline Stable & $\begin{array}{l}\text { stratégies } \\
\text { d'apprentissage }\end{array}$ & $\begin{array}{c}\text { capacités } \\
\text { personnelles } \\
\text { à réaliser la tâche }\end{array}$ & $\begin{array}{l}\text { ressources } \\
\text { extérieures }\end{array}$ & $\begin{array}{l}\text { niveau de difficulté } \\
\text { de la tâche }\end{array}$ \\
\hline Modifiable & $\begin{array}{l}\text { efforts } \\
\text { fournis }\end{array}$ & $\begin{array}{l}\text { forme } \\
\text { physique }\end{array}$ & $\begin{array}{l}\text { perception } \\
\text { de l'enseignant }\end{array}$ & hasard \\
\hline \multicolumn{5}{|c|}{$\begin{array}{l}\text { Certains exemples cités ici peuvent correspondre à plusieurs attributions causales. Il faut, en effet, prendre en compte le } \\
\text { fait que le processus attributionnel est une démarche strictement personnelle - et donc forcément subjective -, qui est } \\
\text { considérablement influencée par les croyances, les valeurs ou encore les expériences antérieures. Par exemple, certains } \\
\text { apprenants considèreront leurs capacités personnelles comme modifiables et non comme stables. }\end{array}$} \\
\hline
\end{tabular}


Selon le psychologue américain, il s'agit d'un facteur de personnalité lié à la perception subjective de l'origine des événements affectant la vie des individus, que ceuxci peuvent rattacher à eux-mêmes (on parle de locus de contrôle interne) ou à des facteurs extérieurs, tel que le hasard (on parle de locus de contrôle externe). Les premiers considèreront ainsi que leurs actions sont susceptibles d'influencer directement le cours de leur vie. Rotter $^{24}$ estime que le locus de contrôle interne a un effet bénéfique sur la motivation et sur la réussite en situation d'apprentissage.

\section{La théorie des buts d'accomplissement}

La théorie des buts d'accomplissement fut développée dans les années quatre-vingt par Dweck et Leggett ${ }^{25}$ dans le cadre conceptuel du besoin d'accomplissement. Cette approche, considérée aujourd'hui comme l'une des théories motivationnelles majeures ${ }^{26}$, vise à comprendre comment les individus cherchent à démontrer leurs compétences. Ainsi, en situation d'apprentissage, il existe trois manières de " construire de la compétence » en référence à trois buts distincts ${ }^{27}$ :

- les buts "de maîtrise " ou "d'apprentissage " génèrent chez l'individu un sentiment de compétence lié au niveau de connaissances atteint ;

- les buts "compétitifs » ou " de performance » situent la compétence dans la comparaison à autrui. Ils sont doubles dans la mesure où deux comportements compétitifs, correspondant à deux buts distincts, caractérisent cette volonté de reconnaissance sociale de la compétence : la recherche de la performance, lorsqu'il s'agit de démontrer que l'on est le meilleur, et l'évitement de l'échec qui incitera l'individu à ne pas entreprendre des tâches dans lesquelles il craint de pouvoir échouer.

L'identification récente de ces trois types de comportements dans le cadre de la théorie des buts d'accomplissement a un intérêt majeur, dans la mesure où il a été démontré par Elliot et Harackiewicz ${ }^{27}$ que les comportements compétitifs de recherche de la performance n'altéraient pas la motivation intrinsèque. Ainsi, la volonté d'apprendre ne semble nullement incompatible avec la recherche de la performance.

En revanche, les comportements d'évitement de l'échec déprécient la motivation intrinsèque. Ils témoignent de la volonté de préserver l'image de soi en évitant les conséquences d'un échec, principalement le fait de paraitre incompétent aux yeux d'autrui. Les apprenants animés par ce type de buts de performance sont susceptibles de mettre en ouvre des stratégies défensives sur- prenantes, lorsqu'elles ne sont pas analysées sous l'angle de la dynamique motivationnelle. Le psychologue américain Covington inscrit ces comportements dans une volonté de maintenir l'estime personnelle au sein d'une société qui valorise la compétence et la réussite. Trois types de stratégies défensives ont été décrit ${ }^{28,29}$ :

- la protection de l'estime de soi consiste pour un sujet à relâcher ses efforts lorsqu'il prend conscience qu'il peut échouer. Un doute persistera ainsi quant à l'attribution de son échec à des causes stables (les capacités intellectuelles) ou modifiables (le manque de travail), préservant de ce fait la perception de compétence ;

- les stratégies d'auto-handicap poussent les individus à créer volontairement des obstacles - réels ou imaginaires - à l'apprentissage, afin de pouvoir invoquer une cause modifiable et contrôlable n'affectant pas la perception de compétence face à un échec; il s'agit, par exemple, de préparer un examen au dernier moment ou de se fixer des objectifs inaccessibles ;

- le pessimisme défensif consiste pour l'apprenant à maintenir de façon irréaliste de faibles attentes de réussite et à minimiser l'importance de la tâche à réaliser.

Si ces stratégies défensives peuvent s'avérer bénéfiques à court terme, du fait qu'elles permettent de préserver l'estime de soi et la perception de compétence, elles s'avèrent délétères lorsqu'elles se répètent, provoquant une diminution du rendement et de la volonté de réussir, ainsi qu'un épuisement émotionnel ${ }^{28,29}$.

La perception qu'ont les étudiants de leur environnement d'apprentissage - davantage que cet environnement en tant que tel - est déterminante dans l'orientation vers les buts de maîtrise ou de performance et dans l'adoption de stratégies défensives. Au cours de leur scolarité, les individus se construisent en effet, lors des interactions qu'ils entretiennent avec les enseignants, leurs camarades et leurs parents, une représentation du système au sein duquel ils évoluent, en fonction des buts poursuivis par celui-ci et de ses normes ${ }^{30}$. Ainsi, le dispositif peut être perçu comme un lieu d'apprentissage, d'acquisition de connaissances ou encore de maintien des compétences; c'est généralement ainsi que l'école primaire est appréhendée par les élèves. Il peut au contraire être perçu comme un lieu d'évaluation et de sélection, tel que le décrivent les élèves et les étudiants de l'enseignement secondaire et supérieur. Cette dernière configuration n'encourage guère la prise de risque, puisque l'objectif est alors de ne pas dévoiler ses lacunes aux enseignants. Certains apprenants vont ainsi avoir tendance à adopter des stratégies défensives. Lorsqu'au 


\section{Références}

contraire, le dispositif éducatif est perçu comme un lieu d'apprentissage, il va favoriser l'adoption de buts de maîtrise. L'investissement dans la tâche et la prise de risque seront favorisés et les échecs seront plus souvent attribués à des causes modifiables ${ }^{30}$.

Dans certains cas, la dissonance perçue entre les buts personnels et les objectifs institutionnels peut affecter le profil motivationnel ${ }^{31}$. Ainsi, l'objectif princeps des facultés de médecine est de former des praticiens compétents; dans certains cas, il peut cependant être concurrencé par des objectifs cachés ou implicites, comme par exemple ceux qui sont liés, en France, à la dimension compétitive qui s'exerce sur les étudiants dans le cadre des épreuves classantes nationales (ECN) et qui peut conduire certaines facultés à privilégier l'objectif de réussite des étudiants aux épreuves, dans le but d'acquérir ou de maintenir un classement national avantageux, au détriment - au moins partiel - de l'objectif de compétence des étudiants diplômés. La confrontation entre des étudiants animés par des buts d'apprentissage et une structure promotrice de la performance inter-institutionnelle est susceptible de provoquer une dissonance à l'origine d'une diminution de la motivation intrinsèque.

\section{La perspective future}

Les buts d'apprentissage et de performance sont, en règle générale, des buts immédiats, relatifs à une tâche en cours. Les buts à long terme et les objectifs professionnels sont tout aussi importants à considérer pour appréhender la question de la dynamique motivationnelle. La théorie de la perspective future - ou perspective d'avenir - fut discutée au début des années quatrevingt-dix par deux psychologues belges, Lens et Decruyenaere ${ }^{32}$. Elle fait référence aux capacités cognitives des individus à anticiper les conséquences à long terme de leurs actions. Dans le domaine de la formation, plus la perspective future de l'apprenant est étendue (autrement dit, plus il arrive à se projeter dans un futur lointain), plus sa motivation sera élevée, dans la mesure où il percevra les activités qui lui sont proposées comme un moyen d'atteindre ses objectifs, leur conférant ainsi une valeur instrumentale, même en l'absence de bénéfices immédiats. Diverses études menées dans les milieux éducatifs conduisirent Lens à conclure que "l'élaboration d'une perspective future au moyen de projets bien structurés, détaillés, étalés sur une longue période de temps et comportant des buts intermédiaires et terminaux, a un effet bénéfique sur la motivation $"^{33}$. Dans le cadre conceptuel de la théorie de l'autodétermination, Vansteenkiste et al. ${ }^{34}$ distinguent les buts à long terme intrinsèques, construits de façon autonome par l'individu et orientés vers son développement autonome (il s'agirait par exemple, pour un étudiant en médecine, de s'investir dans l'apprentissage du module d'infectiologie, car il considère qu'il s'agit d'une discipline qu'il est nécessaire de maitriser pour devenir un bon médecin), des buts à long terme extrinsèques orientés vers la recherche de récompenses extérieures (ces buts caractériseraient l'étudiant s'investissant fortement dans la préparation des ECN afin d'être bien classé et de pouvoir choisir une spécialité rémunératrice). Les auteurs ont mis en évidence que la nature intrinsèque des buts à long terme était associée à une meilleure persistance dans les actions entreprises, à l'utilisation de stratégies d'apprentissage plus efficaces et à l'atteinte d'un niveau de performance plus élevé 34 .

\section{La perception de la valeur de la tâche}

Outre la perception d'autonomie et de compétence, la perception de la valeur de la tâche constitue une troisième dimension essentielle de la dynamique motivationnelle dans une approche socio-cognitive du concept en situation d'apprentissage $^{29,35}$. L'engagement cognitif, la persévérance et le niveau de performance atteint sont directement liés à la valeur perçue de la tâche à accomplir, qui désigne le jugement que porte l'apprenant sur l'intérêt à réaliser une activité, au regard de ses objectifs et de ses attentes.

Wigfield et Eccles ${ }^{36} s^{\prime} y$ intéressèrent en inscrivant leur réflexion dans la lignée du modèle " expectation-valeur " développé notamment par Vroom dans les années soixante et connu sous le nom de «théorie V.I.E. » (valence - instrumentalité - expectation). Celle-ci repose sur la perspective selon laquelle nos comportements ne sont pas uniquement dictés par des besoins ou par des stimuli de l'environnement, mais également par la valeur attribuée à la tâche, par la perception de nos capacités à la réaliser et par les objectifs que nous cherchons à atteindre ${ }^{7}$. Selon Vroom ${ }^{7}$, la motivation à agir est un choix rationnel reposant sur trois facteurs :

- la valence $(\mathrm{V})$ est la valeur positive ou négative attribuée par le sujet aux résultats attendus ;

- l'instrumentalité (I) est la relation perçue par le sujet entre le niveau de performance à atteindre et le résultat attendu ;

- l'expectation (E) désigne les efforts que l'individu se sent capable de déployer afin de parvenir à un objectif donné. Elle dépend de la perception qu'il a de ses chances de réussir ; 
Un individu va ainsi s'investir dans des activités lui permettant d'atteindre des objectifs à l'origine de conséquences qu'il perçoit positivement, dès lors qu'il est convaincu de sa capacité à atteindre ces objectifs et que les résultats obtenus seront la conséquence directe du niveau de performance atteint. Selon cette théorie, la motivation reposerait ainsi en grande partie sur l'attente de résultats positifs.

La valeur de la tâche est étroitement corrélée à la perception de compétence, puisque Wigfield et Eccles ${ }^{36}$ ont mis en évidence que les individus compétents dans une activité ont tendance à la valoriser. Pour expliquer ce lien, les deux auteurs évoquent un mécanisme de protection de l'estime de soi ${ }^{37}$, dont nous avons souligné l'importance à plusieurs reprises.

\section{La théorie de l'auto-efficacité}

Nous avons déjà évoqué l'importance de la perception de compétence dans la dynamique motivationnelle.

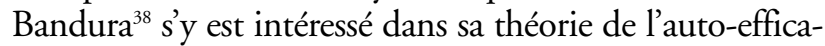
cité. Celle-ci se situe dans le prolongement de la théorie sur l'apprentissage social, dans laquelle le psychologue canadien postule que la conduite humaine, notamment en situation d'apprentissage, repose avant tout sur l'observation et sur l'imitation sélective. Il souligne à ce propos le rôle des influences sociales sur les comportements que l'individu va s'efforcer de sélectionner, selon les conséquences positives ou négatives observées chez ses congénères, et qu'il sera capable d'adapter en fonction des caractéristiques de la situation et de l'environnement ${ }^{39}$.

Dans la théorie de l'auto-efficacité, la perception qu'a l'individu de ses capacités à accomplir une tâche déterminera sa volonté de s'engager dans les actions visant à exécuter cette tâche ; son intérêt intrinsèque pour l'activité sera ainsi plus marqué. Selon Bandura ${ }^{38}$, cette perception s'acquiert par les expériences personnelles (il s'agit notamment des succès déjà obtenus dans la réalisation passée d'une tâche similaire), mais également par l'observation d'autrui ; dans cette approche, "l'expérience vicariante" constitue une ressource importante qui influencera considérablement la perception d'auto-efficacité. Cette dernière peut être influencée par des croyances populaires, telle que celle qui prétend que la chirurgie est un « métier d'homme ", affectant d'emblée la perception de compétence des étudiants du sexe opposé vis-à-vis de cette discipline.

Les théories alimentant notre réflexion sur le dyna- mique motivationnelle sont nombreuses. Elles nous permettent d'envisager un certain nombre d'actions susceptibles d'influencer positivement le niveau d'investissement et la persévérance des apprenants dans les tâches entreprises, notamment en encourageant les comportements fortement autodéterminés, caractéristiques de la motivation intrinsèque et extrinsèque par régulation identifiée.

Avant d'aborder cette partie, il nous semble intéressant de faire référence aux travaux importants sur la motivation réalisés dans le domaine de la formation des professionnels de santé.

\section{La motivation en formation initiale et continue des professionnels de santé: à propos de quelques études}

Divers travaux de recherche ont été menés afin d'établir le profil motivationnel des étudiants des filières de santé.

Sobral ${ }^{40}$ a questionné 297 étudiants au début de leur deuxième année de médecine. Leur profil motivationnel s'est révélé fortement autodéterminé, puisque les réponses explorant la motivation intrinsèque et extrinsèque par régulation identifiée ont été les plus souvent citées. Il existe un lien entre le niveau d'autodétermination et les stratégies d'apprentissage utilisées : les étudiants intrinsèquement motivés engagent un travail de réflexion bien plus important au regard des tâches à accomplir que les étudiants agissant sous l'influence de facteurs extérieurs, voire amotivés, qui se contentent d'un apprentissage superficiel basé sur la mémorisation du contenu des cours. Dans ce domaine, plusieurs études ont mis en évidence qu'un apprentissage en profondeur et l'usage de stratégies métacognitives étaient associées à de meilleures performances ${ }^{41,42}$. Le suivi de la cohorte sur une durée de deux ans a ainsi permis de corréler un niveau élevé d'autodétermination à une meilleure réussite aux examens ${ }^{40}$. Enfin, la volonté de poursuivre les études et de réussir l'année universitaire était également plus marquée chez les apprenants intrinsèquement motivés.

Perrot et al. ${ }^{43}$ ont étudié la motivation de 252 étudiants de première année de médecine, de pharmacie et de soins infirmiers au regard de la théorie des buts d'accomplissement. D'après leurs travaux, les buts de mầtrise caractérisent le plus souvent le profil motivationnel des populations étudiées, en particulier chez les étu- 


\section{Références}

diants en médecine et en soins infirmiers. Les auteurs ont également mis en évidence le fait que les étudiants animés par des buts de maîtrise mettaient préférentiellement en œuvre des stratégies d'apprentissage métacognitives ; ils sont capables de mener une réflexion sur les méthodes et les processus intellectuels qu'ils mobilisent face à une tâche à accomplir, afin de privilégier les plus efficaces. Les étudiants des filières de santé ont plutôt un locus de contrôle interne, ce qui tend à confirmer la corrélation de ce facteur de personnalité aux buts de maîtrise ${ }^{44}$.

Dans ce champ théorique des buts d'accomplissement, Gardner ${ }^{45}$ a démontré que des formations courtes exposant les différences entre les buts de performance et les buts de maitrise étaient susceptibles d'influencer positivement le type de buts poursuivi par des étudiants en deuxième année de soins infirmiers.

Misch ${ }^{46}$ inscrit quant à lui sa réflexion sur la motivation des étudiants en médecine dans le cadre théorique de l'andragogie. Il insiste d'une part, sur le caractère évolutif de la motivation et d'autre part, sur ses liens avec l'environnement d'apprentissage, notamment les principes d'évaluation des apprentissages en œuvre au sein des facultés de médecine - par le biais des examens -, qui tendent, selon l'auteur, à décourager toute prise de risque.

Ces deux aspects de la motivation ont été appréhendés dans le domaine de la formation des étudiants en médecine par deux travaux de recherche distincts.

Barker et Olson ${ }^{47}$ se sont intéressés à l'évolution du profil motivationnel des étudiants en médecine au cours de la première année et à son influence sur les stratégies d'apprentissage utilisées. Dans cette perspective, 64 étudiants furent soumis à un questionnaire explorant différents aspects de la motivation, lors de leur admission en première année de médecine et à l'issue de celle-ci. Le niveau de motivation intrinsèque, la perception de la valeur de la tâche et du contrôle, ainsi que l'usage de stratégies d'apprentissage métacognitives furent tous affectés négativement au cours de la première année. La motivation extrinsèque et la perception de compétence demeurèrent quant à elles inchangées. Barker et Olson attribuent la diminution de la motivation intrinsèque aux difficultés éprouvées par les étudiants à mettre en lien le programme de la première année de médecine et leur futur métier, ce qui confirme l'importance de la perception de la valeur de la tâche et de la perspective d'avenir dans la dynamique motivationnelle. Ils estiment, par ailleurs, que les changements de stratégies d'apprentissage sont liés à l'environnement compétitif qui incite les étudiants à la mémorisation plutôt qu’à la réflexion, dans la perspective d'obtenir de bons résultats lors des épreuves certificatives. Enfin, conformément aux approches théoriques exposées précédemment, les tests de corrélation réalisés par rapport aux moyennes pondérées des notes obtenues par les étudiants lors de leurs examens mirent en évidence qu'un niveau élevé de motivation intrinsèque et de perception de compétence étaient indépendamment corrélés à de meilleurs résultats. Il s'agit là, selon les auteurs, des deux dimensions majeures de la motivation à prendre en compte par les responsables pédagogiques des facultés de médecine.

Concernant l'influence du système d'évaluation sur la dynamique motivationnelle, O'Neill et al. ${ }^{48}$ ont interrogé 1290 britanniques étudiants en médecine afin de recueillir leur opinion sur l'intérêt du dispositif qui permet d'être diplômé « avec les honneurs ", grâce à l'accumulation de points attribués tout au long du cursus en fonction des notes obtenues aux examens. Lanalyse des résultats a montré que le système était soutenu par $75 \%$ de la population sondée, mais que plus les étudiants étaient à un niveau avancé du cursus, plus ils le désapprouvaient et le considéraient comme une source de démotivation. Les auteurs ont, par ailleurs, mis en évidence une corrélation positive entre le nombre de points accumulé par les étudiants et leur niveau d'approbation du système au regard de son impact sur la motivation. Ces résultats intéressants vont dans le sens de la réflexion menée par Patel et David ${ }^{49}$ dans un article intitulé "Everybody has won, and all must have prizes". Les deux auteurs estiment, en effet, que les récompenses extrinsèques créent artificiellement une notion de succès, qui n'existe que dans son opposition à l'échec et qui est susceptible de diminuer la motivation, dans la mesure où le succès n'est pas certain. Ainsi, un système basé sur les récompenses engendrerait chez ceux qui «ne gagnent pas " un sentiment d'échec, une diminution de l'estime personnelle et de la perception de compétence, qui, conformément à la théorie de l'évaluation cognitive de Deci et Ryan" ${ }^{90}$, déprécient la motivation intrinsèque à accomplir une tâche, même lorsque l'étudiant en est capable. Patel et David ${ }^{49}$ rappellent en outre que le système a tendance à générer des locus de contrôle externes, préjudiciables à l'apprentissage.

Finalement, le principe des mentions ne profiterait qu'à ceux qui pensent pouvoir en obtenir et altérerait la motivation des autres. O'Neill et al. ${ }^{48}$ soulignent à ce 
propos que seuls $10 \%$ de leurs étudiants sont diplômés "avec les honneurs " et que les deux-tiers n'auront jamais de point.

Certains dispositifs pédagogiques revendiquent explicitement un impact positif sur la motivation intrinsèque. C'est notamment le cas des cursus centrés sur l'apprentissage par problème $(\mathrm{APP})^{50}$. Une étude réalisée à ce propos en 1986 par De Voler et al..$^{51}$ démontra que l'APP était susceptible d'engendrer des comportements d'apprentissage intrinsèquement motivés, en raison de la curiosité suscitée à propos du sujet d'étude, de la dimension de défi associée à la nécessité de résoudre un problème - en l'absence de récompenses tangibles - et de l'impact positif de la dynamique de groupe. Une étude ultérieure ${ }^{52}$ mit toutefois en évidence que des déséquilibres générés au sein des groupes d'APP par des comportements inadaptés (étudiants en retrait ou dominants, absentéisme) étaient susceptibles d'altérer le niveau motivationnel. Il en est de même lorsque l'apprenant est confronté à des étudiants passifs n'ayant pas fait l'effort de préparer la séance ${ }^{53}$. Hendry et al. ${ }^{54}$ ont à ce propos démontré un bénéfice motivationnel des petits groupes de travail, constitués spontanément par les étudiants et se réunissant de façon hebdomadaire, afin d'apporter des clarifications sur les thèmes abordés dans le cadre des séances d'APP.

Dans un cadre expérimental, White ${ }^{55}$ a comparé les profils motivationnels d'étudiants en médecine américains évoluant dans un dispositif traditionnel avec ceux bénéficiant d'une approche par problème. L'étude révéla que le profil motivationnel précédant l'admission en faculté de médecine était régulé dans les deux cas par des facteurs externes, du fait du processus de sélection nécessitant l'obtention de notes élevées. Leur motivation a ensuite évolué de façon distincte en fonction du type de dispositif pédagogique en œuvre au sein de l'université : les étudiants bénéficiant de l'APP soulignèrent les difficultés liées à la transition entre un enseignement traditionnel et l'usage de cette approche pédagogique. Une perception positive d'autonomie et de contrôle sur leur formation s'est affirmée à l'issue du premier semestre de la première année, ainsi que la mise en œuvre de stratégies d'apprentissage métacognitives. Cette évolution s'est accompagnée d'une plus grande motivation intrinsèque en raison du développement de buts d'apprentissage. Les étudiants inscrits dans un cursus traditionnel ne perçurent pas les mêmes difficultés transitionnelles que les précédents, en raison du caractère similaire des approches pédagogiques utilisées. De ce fait, ils ne manifestaient pas le besoin de faire usage de stratégies d'apprentissage appronfondies et se contentaient de mémoriser le contenu important de leurs cours, dans la perspective d'obtenir de bonnes notes aux examens. Ce manque d'autonomie et la perception d'un contrôle exercé par la faculté sur leur apprentissage généra des difficultés lors de leur passage en troisième année (marquée par le début des stages cliniques), que ne perçurent pas les étudiants des cursus innovants. Une étude similaire réalisée en $1994^{56}$ et comparant une cohorte de 137 étudiants bénéficiant d'un cursus innovant avec 149 étudiants inscrits dans un cursus traditionnel était parvenue à des conclusions similaires au regard du bénéfice motivationnel de l'APP.

Dans le même registre, McLean ${ }^{57}$ s'est intéressée à des étudiants en échec à l'issue de la deuxième année de médecine, qui avaient le choix de poursuivre leurs études au sein du même dispositif, ou bien de s'engager dans un cursus basé sur une approche par problème. Les étudiants ayant opté pour la seconde possibilité, outre un niveau de satisfaction plus élevé, considéraient l'APP comme une activité intrinsèquement motivante pour l'apprentissage.

Dans le domaine paramédical, Hwang et $\mathrm{Kim}^{58}$ ont comparé le niveau motivationnel et les comportements d'apprentissage de 35 étudiants inscrits en deuxième année d'un cursus en soins infirmiers basé sur une approche par problème et de 36 étudiants d'une formation traditionnelle. Le premier groupe manifestait un niveau de motivation intrinsèque supérieur aux autres, que les auteurs attribuèrent à une plus grande autonomie au sein du dispositif. Des résultats similaires furent mis en évidence dans d'autres travaux de recherche réalisés sur ce thème $\mathrm{e}^{59-61}$. Hwang et $\mathrm{Kim}^{58}$ soulignent toutefois que les comportements d'apprentissage d'un certain nombre d'étudiants étaient dirigés à la fois par des facteurs intrinsèques et extrinsèques, comme l'avait démontré Sobral à propos des étudiants en médecine ${ }^{40}$. En revanche, contrairement à d'autres études ${ }^{58}$, aucune différence significative ne fut observée quant aux stratégies d'apprentissage utilisées.

Nous avons souhaité explorer plus précisément les raisons pour lesquelles l'APP constituait une activité "motivante ". Dans un travail de synthèse réalisé en 2003 à ce sujet, Pedersen ${ }^{62}$ souligne que l'étudiant confronté à une situation problématique prend conscience de son manque de connaissances. Il va donc s'engager dans une démarche personnelle et autorégulée de recherche d'informations dans la perspective d'une 


\section{Références}

résolution du problème posé. Cette perception majorée d'autonomie - au regard des cursus traditionnels dans lesquels ce sont les enseignants qui déterminent les objectifs pédagogiques et qui régulent le rythme d'apprentissage des étudiants (en imposant la fréquence des cours et la nature de leur contenu) - est à l'origine d'un effet bénéfique sur la motivation intrinsèque. Le sentiment de contrôle sur son propre apprentissage est renforcé par la perception de liberté quant au choix des tâches à accomplir au sein du groupe, afin de contribuer à résoudre le problème. L'auteur souligne, par ailleurs, que la dimension ludique de l'activité, son caractère novateur au regard des techniques pédagogiques traditionnelles et l'absence de récompenses extrinsèques tangibles ont également un effet bénéfique sur le profil motivationnel. L'APP permet en outre d'augmenter la perception de la valeur de la tâche au regard de l'activité professionnelle future des étudiants, dans la mesure où des liens sont établis entre les sciences fondamentales et les disciplines cliniques. Un autre effet positif des approches par problème concerne l'augmentation de la perception de compétence liée à la résolution de tâches stimulantes associées à une dimension de défi.

$\mathrm{Au}$ regard des approches théoriques exposées précédemment, il est aisé de comprendre que l'association d'un sentiment d'efficacité et d'autonomie renforce l'estime personnelle des apprenants et réduit le risque qu'ils adoptent des stratégies défensives. La prise de risque est d'ailleurs encouragée et valorisée par les tuteurs qui encadrent les séances d'APP. Enfin, la dimension collaborative de la démarche pédagogique et l'observation de ses pairs dans des tâches d'apprentissage - et en particulier dans leur réussite - est de nature, au regard de la théorie de l'apprentissage social et de la théorie de l'auto-efficacité de Bandura, de majorer la motivation à accomplir ces mêmes activités.

Soulignons à ce sujet que si l'APP stimule les comportements d'apprentissage intrinsèquement motivés, ces derniers sont également une condition essentielle de l'efficacité de l'approche pédagogique au regard des effets qu'elle provoque sur la dynamique de groupe et sur le processus d'apprentissage ${ }^{63-66}$.

La question de la motivation des professionnels de santé en formation continue a été étudiée en 2007 par Hopstock $^{67}$. Trois cent soixante-et-un personnels hospitaliers -majoritairement des infirmiers - furent questionnés au début d'une séance d'apprentissage consacrée à la prise en charge de l'arrêt cardiaque. La perception de la valeur de la tâche était significativement plus élevée chez les professionnels de santé travaillant au contact de malades, en particulier dans les secteurs à haut risque d'arrêt cardiaque dont les soignants manifestaient également un sentiment élevé d'efficacité personnelle. Conformément à l'approche théorique de Wigfield et $\mathrm{Eccles}^{37}$, une corrélation positive a été mise en évidence entre la perception de compétence et la perception de la valeur de la tâche. Cette dernière était également supérieure chez les personnes ayant préalablement bénéficié d'une séance d'information soulignant l'importance de maîtriser les gestes de réanimation cardio-pulmonaire et présentant le programme de la formation, ce qui a permis à un certain nombre de stagiaires de prendre conscience de l'écart entre leurs connaissances actuelles et celles visées par la formation. Enfin, la perception de la valeur de la tâche était plus élevée chez les stagiaires ayant décidé volontairement et sans contrainte extérieure de participer à l'activité, ce qui souligne l'importance de la perception d'autonomie et de contrôle. Pour l'auteur, le maintien d'un niveau élevé de perception de la valeur de la tâche à distance de la formation est difficile à obtenir, ce qui pourrait expliquer l'altération rapide des connaissances, y compris chez les personnes manifestant une perception élevée de compétence.

Au total, plusieurs travaux de recherche conduits en milieu authentique, réalisés auprès de professionnels de santé en formation initiale ou continue, confirment la pertinence des théories présentées, dans le champ disciplinaire des sciences de la santé. Il est dès lors possible de s'appuyer sur de tels cadres conceptuels et sur de tels résultats pour proposer quelques pistes de réflexion quant aux actions à mettre en œuvre pour favoriser la dynamique motivationnelle des étudiants et des professionnels de santé en formation.

\section{Les implications pratiques des théories motivationnelles sur la formation initiale et continue des professionnels de santé}

Les actions destinées à modifier le profil motivationnel des étudiants avant leur engagement dans le dispositif éducatif sont réduites. Les perspectives d'action sur l'environnement, sur les tâches d'apprentissage et sur l'apprenant sont, en revanche, beaucoup plus vastes, une fois l'entrée en formation effective. Nous les présentons en lien avec les théories motivationnelles décrites dans la première partie du document. 


\section{Augmenter la perception de la valeur de la tâche}

Au regard des approches théoriques exposées précédemment $^{7,25-27,32,36}$, une perception élevée de la valeur de la tâche et des perspectives futures étendues sont associées à des comportements intrinsèquement motivés.

Il est ainsi nécessaire de définir explicitement des buts d'apprentissage en termes d'objectifs éducationnels et de compétences visées. Les actions possibles dans ce domaine se situent à différents niveaux :

- il s'agit d'abord du niveau macrocontextuel des objectifs institutionnels qui doivent mettre en valeur la finalité de la formation dans ses dimensions éducatives, plutôt que sous l'angle de la recherche de performance normative ;

- les buts d'apprentissage doivent également être définis à un niveau microcontextuel. Il s'agit précisément d'exposer les objectifs des séquences d'apprentissage, en valorisant leurs liens avec l'activité professionnelle active ou future des apprenants. Dans ce registre, les objectifs définis doivent permettre à l'étudiant de s'engager cognitivement dans la résolution de tâches dont le niveau de complexité ne doit être ni trop élevé (cela peut mener à l'abandon), ni trop bas (les objectifs faciles à atteindre provoquent un désintérêt pour l'activité $)^{20}$;

- il s'agit enfin du niveau individuel, dans la mesure où une concordance entre les objectifs institutionnels et les attentes des apprenants aura un impact positif sur la motivation. Il convient donc, dans la mesure du possible, d'explorer les objectifs personnels.

\section{Augmenter la perception d'autonomie et de contrôle}

Les théories formulées à cet égard montrent un effet bénéfique de la perception d'autonomie et de contrôle sur la dynamique motivationnelle ${ }^{19,22,24}$.

Dans cette perspective, sous certaines conditions et selon le contexte, il peut être souhaitable de laisser à l'apprenant des libertés quant à certains choix concernant, par exemple, les ressources didactiques utilisables, les terrains de stage, les horaires ou encore les stratégies d'enseignement et d'apprentissage. Dans ce cadre, les dispositifs intégrant des pratiques d'autoévaluation des apprenants ont également une influence positive sur la motivation. Une autonomie totale n'est bien entendu pas envisageable, en particulier au regard des contraintes institutionnelles (obligations en matière de formation, impératifs de validation d'unités d'enseigne- ment et de certification de compétences, etc.) et pourrait même s'avérer préjudiciable, notamment en formation initiale, puisque des travaux de recherche ${ }^{54}$ réalisés à propos de l'APP ont démontré les difficultés d'adaptation initiale des étudiants confrontés brutalement à un environnement au sein duquel ils disposent d'une forte autonomie, sans y avoir été préparés. Il faut garder à l'esprit que l'autonomie d'apprentissage est d'abord une finalité visée chez les étudiants, s'inscrivant dans le cadre d'exigences à atteindre à l'issue de programme recourant à des dispositifs pédagogiques tels que l'APP ; il serait erroné de postuler que les étudiants vont manifester cette caractéristique d'emblée, du seul fait qu'ils sont exposés au dispositif. Il convient donc de mettre à leur disposition une assistance adaptée au niveau d'autonomie qu'ils ont acquis, sous peine d'induire une démotivation liée à la perception de tâches qui seraient trop difficiles à maîtriser en autonomie totale.

\section{Augmenter la perception de compétence et d'efficacité personnelle}

Plusieurs approches théoriques ${ }^{21,38}$ soulignent que l'intérêt intrinsèque pour une activité est renforcé par la perception de compétence de l'individu au regard des tâches à accomplir.

Le rôle du feeback et du tutorat est ici fondamental. Informer l'apprenant sur les stratégies d'apprentissage, le renseigner sur sa progression, l'encourager lorsqu'il est performant, le rassurer sur ses capacités lorsqu'il est défaillant sont autant d'actions qui ont un impact positif majeur sur la motivation.

\section{Privilégier l'usage de méthodes d'enseigne- ment intrinsèquement motivantes}

Les études réalisées à propos de l'APP mettent en évidence un impact positif du dispositif sur la motivation, en raison de l'implication active de l'apprenant dans la construction de ses connaissances, de sa perception majorée d'autonomie, de la dynamique de groupe, de la contextualisation des problèmes exposés et de la dimension de défi associée à l'activitét ${ }^{61}$.

Ces travaux permettent d'envisager que d'autres méthodes actives d'enseignement remplissant ces conditions d'apprentissage sont susceptibles de motiver intrinsèquement les étudiants et les stagiaires de la formation continue. Il s'agit, par exemple, des méthodes de découverte, de la méthode des cas ou encore de la simulation. 


\section{Références}

\section{Favoriser l'usage de facteurs extrinsèques n'altérant pas la motivation intrinsèque}

Plusieurs auteurs ${ }^{21}$ ont mis en évidence le caractère potentiellement préjudiciable - en termes de motivation - des récompenses externes, en particulier lorsqu'elles sont tangibles et attribuées dans un environnement perçu par l'apprenant comme " contrôlant ».

Au regard de cette approche, nous avons déjà souligné l'importance du "feedback » verbal. Il est cependant difficilement envisageable de restreindre l'évaluation des apprenants - en particulier en formation initiale - à ce seul outil, comme en témoigne l'omniprésence des systèmes de notation dans le cadre des dispositifs d'évaluation sommative et normative. Il est, en revanche, possible de limiter l'impact motivationnel négatif des examens et des contrôles de connaissances au regard de leur contenu. Lors de ces épreuves, il est ainsi préférable d'évaluer si l'apprenant a atteint l'ensemble des objectifs majeurs de la formation. Soumettre l'individu à des questions ne portant que sur des aspects spécifiques et restrictifs du programme, en particulier lorsqu'elles cherchent à explorer la mémorisation d'éléments de détail du contenu des enseignements dispensés, est perçu, au regard des conceptions théoriques admises ${ }^{25-27,30}$, comme une activité contrôlante, destinée à satisfaire des objectifs de sélection plutôt que d'évaluation des apprentissages dans une perspective d'efficacité professionnelle. Une telle approche docimologique comporte donc de nombreuses limites au regard de la dynamique motivationnelle. Les systèmes de mention et autres dispositifs de valorisation de l'excellence sont par ailleurs préjudiciables pour la plupart des apprenants ${ }^{48,49}$. Il convient, pour les responsables pédagogiques des facultés et des structures de formation, d'évaluer dans ce cadre, comme dans les précédents, le rapport bénéfices-risques de tels systèmes, au regard des objectifs poursuivis par l'institution. Il est fortement souhaitable qu'il existe une congruence et une cohérence entre les principes directeurs retenus par l'institution concernant les formats d'intervention pédagogique et ceux qui sous tendent les procédures d'évaluation mises en œuvre.

\section{Conclusion}

Dans un environnement d'apprentissage impliquant de façon croissante l'apprenant dans la construction de ses connaissances et de ses compétences, la question de la motivation est centrale. Nous avons abordé ce thème à deux niveaux d'analyse, théoriquement distincts, mais pratiquement indissociables :
- celui des motifs d'engagement en formation, qui nous a conduit à identifier des facteurs intrinsèques et extrinsèques de motivation, qui different par le niveau d'autodétermination perçu par l'individu ;

- celui de la dynamique motivationnelle, qui nous a permis de formuler des propositions concrètes d'actions sur l'individu et sur son environnement, afin de lui permettre d'adopter des comportements d'apprentissage durables et efficaces.

La motivation n'est bien entendu par le déterminant unique de la réussite. Elle en constitue toutefois une dimension majeure, à laquelle il devient nécessaire de porter une attention particulière au regard d'un environnement d'apprentissage en mutation.

\section{Remerciements}

Les auteurs remercient très vivement le rédacteur en chef, le comité de rédaction et les lecteurs-experts, dont la pertinence des remarques a été fondamentalement contributive à la rédaction de la version finale de l'article.

\section{Contributions}

Les auteurs ont conjointement mené des discussions sur la structuration du champ théorique et du cadre d'analyse initiaux et sur l'élaboration de l'investigation conceptuelle. Thierry Pelaccia a rédigé une première version de l'article qui a été révisée avec les coauteurs à l'aide des commentaires formulés par le comité de rédaction et par les lecteurs-experts. La version finale du manuscrit a été approuvée par l'ensemble des auteurs. 


\section{Références}

1. Vallerand RJ, Thill EE. Introduction au concept de motivation. In : Vallerand RJ, Thill EE (eds). Introduction à la psychologie de la motivation. Laval (Québec) : Éditions Etudes vivantes, 1993:3-39.

2. Bourgeois E. Apprentissage, motivation et engagement en formation. Education permanente 1993;136:1019 .

3. Carré P. De la motivation à la formation. Paris : Éditions L'Harmattan, 2001.

4. Bourgeois E, Chapelle G. Apprendre et faire apprendre. Paris: Presses universitaires de France, 2006.

5. Feertchak H. Motivations, valeurs et horizon temporel. Éducation permanente 1998;136:183-90.

6. Nuttin J. Théorie de la motivation humaine. Paris : Presses universitaires de France, 1980.

7. Fenouillet F. La motivation. Paris : Editions Dunod, 2003.

8. Bouffard T, Marine C, Chouinard R. Interdépendance des caractéristiques individuelles et contextuelles dans la motivation à apprendre. Revues des sciences de l'éducation 2004;30:3-8.

9. Vallerand RJ, Blanchard C. Éducation permanente et motivation : contribution du modèle hiérarchique de la motivation intrinsèque et extrinsèque. Éducation permanente 1993;13:15-35.

10. Deci EL, Ryan RM. Intrinsic Motivation and SelfDetermination in Human Behavior. New York : Editions Plenum Press, 1985.

11. Nuttin J. Développement de la motivation en formation. Education permanente 1986;88:97-110.

12. Famose JP, Fleurance P, Touchard Y. L'apprentissage moteur, rôle des représentations. Paris : Éditions EPS, 1991.

13. Charlot B. Du rapport au savoir: éléments pour une théorie. Paris : Editions Anthropos, 1999.

14. Ryan RM, Deci EL. Intrinsic and Extrinsic Motivations : Classic Definitions and New Directions. Contemporary Educational Psychology 2000;25:54-67.
15. Muchielli A. Les motivations. Paris : Presses universitaires de France, 2006.

16. Vallerand RJ, Reid G. On the Causal Effects of Perceived Competence on Intrinsic Motivation : A Test of Cognitive Evaluation Theory. Journal of Sport Psychology 1984;6:94-102.

17. Ryan RM. Psychological Needs and the Facilitation of Integrative Processes. J Pers 1995;63:397-427.

18. Vallerand RJ, Blais MR, Briere NM, Pelletier. Construction et validation de l'Échelle de Motivation en Éducation (EME). Revue canadienne des sciences du comportement 1989;21:323-49.

19. Deci EL, Koestner R, Ryan RM. Extrinsic Rewards and Intrinsic Motivation in Education : Reconsidered Once Again. Review of Educational Research 2001;1:1-27.

20. Paris SG, Turner JC. Situated Motivation. In : Pintrich, PR, Brown DR, Weinstein CE (eds). Student Motivation, Cognition, and Learning. Hillsdale (New-Jersey) : Lawrence Erlbaum Associates, 1994:213-37.

21. Deci EL, Koestner R, Ryan RM. A Meta-Analytic Review of Experiments Examining the Effects of Extrinsic Rewards on Intrinsic Motivation. Psychological Bulletin 1999;125:627-68.

22. Weiner B. Motivation From an Attribution Perspective and the Social Psychology of Perceived Competence. In : Elliot AJ, Dweck CS (eds.). Handbook of Competence and Motivation. NewYork: Guilford Press, 2005:73-84.

23. Vianin P. La motivation scolaire : comment susciter le désir d'apprendre ? Bruxelles : Editions de De Boeck, 2006.

24. Rotter JB. Generalized Expectancies for Internal Versus External Control of Reinforcement. Psychological Monograph 1966;80:1-28.

25. Dweck CS, Leggett E. A Social-Cognitive Approach to Motivation and Personality. Psychol Rev 1988;95:256-73. 


\section{Références}

26. Elliot AJ, Dweck CS. Competence as the Core of Achievement Motivation. In : Elliot AJ, Dweck CS (eds.). Handbook of Competence and Motivation. New-York: Guilford Press, 2005:3-13.

27. Elliot AJ, Harackiewicz J. Approach and Avoidance Achievement Goals and Intrinsic Motivation : A Mediational Analysis. Personality and Social Psychology 1996;70:461-75.

28. Covington MV. Goal Theory, Motivation, and School Achievement: An Integrative Review. Annual Psychological Review 2000;51:171-200.

29. iau R. La motivation en contexte scolaire. Bruxelles: Éditions de De Boeck (2e édition, 1998.

30. Crahay M. Psychologie de l'éducation. Paris : Presses universitaires de France, 1999.

31. Mann KV. Motivation in Medical Education : How Theory Can Inform Our Practice. Acad Med 1999;74:237-9.

32. Lens W, Decruyenaere M. Motivation and DeMotivation in Secondary Education : Student Characteristics. Learning and Instruction 1991;14559.

33. Lens W. Motivation and Learning (rapport de recherche). Centre de recherche sur la motivation. Université de Louvain (Belgique), 1991.

34. Vansteenkiste M, Simons J, Soenens B, Lens W. How to Become a Persevering Exerciser? Providing a Clear, Future Intrinsic Goal in an Autonomy-Supportive Way. Journal of Sport and Exercise Psychology 2004;26:23249.

35. Viau R, Bouchard J. Validation d'un modèle de dynamique motivationnelle auprès d'élèves du secondaire. Revue canadienne de l'éducation 2000 ;25:16-26.

36. Wigfield A, Eccles JS. Expectancy-Value Theory of Achievement Motivation. Contemporary Educational Psychology 2000;65:68-81.

37. Wigfield A, Eccles JS. The Development of Achievement Task Values : A Theoritical Analysis. Developmental Review 1992;12:265-310.

38. Bandura A. Auto-efficacité : le sentiment d'efficacité personnelle. Bruxelles : Éditions de De Boeck Université, 2003.
39. Bandura A. L'apprentissage social. Bruxelles : Éditions Mardaga, 1976.

40. Sobral DT. What Kind of Motivation Drives Medical Students' Learning Quests? Med Educ 2004;8:950-7.

41. Zeegers P. Approaches to Learning in Science: A Longitudinal Study. Br J Educ Psychol 2001;7:11532.

42. Dunning D, Johnson K, Ehrlinger J, Kruger J. Why People Fail to Recognize Their Own Incompetence. Current Directions in Psychological Science 2003 ; 12:837.

43. Perrot LJ, Deloney LA, Hastings JK, Savell S, Savidge M. Measuring Student Motivation in Health Professions' Colleges. Adv Health Sci Educ 2001;6:193-203.

44. Seifert TL, O'Keefe BA. The Relationship of Work Avoidance and Learning Goals to Perceived Competence, Externality and Meaning. Br J Educ Psychol 2001;71:81-92.

45. Gardner EA. Instructing in Mastery Goal Orientation : Developing Problem Solving and Persistence for Clinical Settings. Journal of Nursing Education 2006 ;45:343-7.

46. Misch DA. Andragogy and Medical Education : Are Medical Students Internally Motivated to Learn? Adv Health Sci Educ 2002 ; 7:153-60.

47. Barker JR, Olson JP. Medical Students' Learning Strategies: Evaluation of First Year Changes. 1999. [Online]. Disponible sur : http://www.msstate.edu/org/MAS/ ejour2.htmll

48. O'Neill P, Baxter CM, Morris J. Does Awarding a Medical Degree With Honors Act as a Motivator or Demotivator to Student Learning? Med Educ 1999;33:566-71.

49. Patel L, David TJ. Everybody Has Won, and All Must Have Prizes. Lancet 1996 ;348:1497.

50. Barrows HS, Tamblyn RM. Problem-Based Learning : An Approach to Medical Education. New-York : Springer Publishing Company, 1980.

51. DeVolder ML, Schmidt HG, Moust JHC, Degrave WS. Problem-Based Learning and Intrinsic Motivation. In: Van Der Berchen JHC, Bergen TCM, De Bruyn EEI (eds.). Achivement and Task Motivation. Lisse (PaysBas):Swets and Zeitlinger, 1986:23-5. 
52. Hendry GD, Ryan G, Harris J.Group Problems in Problem-Based Learning. Med Teach 2003 ;25:60916.

53. De Grave WS, Dolmans DHJM, Van Der Vleuten CPM. Students Perceptions About the Occurrence of Critical Incidents in Tutorial Groups. Med Teach 2001;23:49-54.

54. Hendry GD, Hyde SJ, Davy P. Independent Study Groups. Med Educ 2005;39:672-9.

55. White CB. Smoothing Out Transitions : How Pedagogy Influences Medical Students' Achievement of Self-Regulated Learning Goals. Adv Health Sci Educ 2007;12:279-97.

56. Bligh J, Lloyd-Jones G, Smith G. Early Effects of a New Problem-Based Clinically Oriented Curriculum on Students' Perceptions of Teaching. Med Educ 2000;34:487-9.

57. McLean M. A Comparison of Students Who Chose a Traditional or a Problem-Based Learning Curriculum After Failing Year 2 in the Traditional Curriculum : A Unique Case Study at the Nelson R. Mandela School of Medicine. Teach Learn Med 2004;16:301-3.

58. Hwang SY, Kim MJ. A Comparison of ProblemBased Learning and Lecture-Based Learning in an Adult Health Nursing Course. Nurse Education Today 2006;26:315-21.

59. Cooke M, Moyle K. Students' Evaluation of ProblemBased Learning. Nurse Education Today 2002;22:330-9.
60. Williams B. Self-Direction in a Problem-Based Learning Program, Nurse Education Today 2004;19:659-67.

61. Tiwari A, Chan S, Wong E, Wong D, Chui C, Wong A, Patil N. The Effect of Problem-Based Learning on Students' Approaches to Learning in the Context of Clinical Nursing Education. Nurse Education Today 2006;26:430-8.

62. Pedersen S. Motivational Orientation in a ProblemBased Learning Environment. Journal of Interactive Learning Research 2003;14:51-77.

63. Pintrich PA. The Role of Motivation in Promoting and Sustaining Self-Regulated Learning. International Journal of Science Education 1999;31:459-70.

64. Dolmans $D H$, Wolfhagen $I H$, van der Vleuten $C P$, Wijnen WH. Solving Problems With Group Work in Problem-Based Learning: Hold on to the Philosophy. Med Educ 2001;35:884-9.

65. De Grave WS, Dolmans DHJM, Van Der Vleuten CPM. Students Perspectives on Critical Incidents in the Tutorial Group. Advances in Health Science Education 2002;7: 201-9.

66. Das Carlo M, Swadi H, Mpofu D. Medical Student Perceptions of Factors Affecting Productivity of Problem-Based Learning Tutorial Groups : Does Culture Influence the Outcome? Teach Learn Med 2003; 15:59-64.

67. Hopstock LA. Motivation and Adult Learning : A Survey Among Hospital Personnel Attending a CPR Course. Resuscitation 2007;76:425-30.

Manuscrit reçu le 4 novembre 2007 ; commentaires éditoriaux formulés aux auteurs le 3 décembre 2007 et le 25 mars 2008; accepté pour publication le 2 avril 2008. 